\title{
Three Rett patients with both MECP2 mutation and 15q11-13 rearrangements
}

\author{
Ilaria Longo ${ }^{1}$, Luisa Russo ${ }^{2}$, Ilaria Meloni ${ }^{1}$, Iolanda Ricci $^{2}$, Francesca Ariani ${ }^{1}$, \\ Chiara Pescucci $^{1}$, Carmela Tiziana Giordano ${ }^{2}$, Roberto Canitano ${ }^{3}$, Giuseppe Hayek ${ }^{3}$, \\ Michele Zappella ${ }^{3}$, Giovanni Neri ${ }^{2}$, Alessandra Renieri ${ }^{1}$ and Fiorella Gurrieri*,2 \\ ${ }^{1}$ Medical Genetics, University of Siena, Italy; ${ }^{2}$ Medical Genetics, Università Cattolica del Sacro Cuore, Roma, Italy; \\ ${ }^{3}$ Neuropsichiatria Infantile, Azienda Ospedaliera Senese, Siena, Italy
}

\begin{abstract}
Autism and Rett syndrome, a severe neurological disorder with autistic behavior, are classified as separate disorders on clinical and etiological ground. Rett syndrome is a monogenic X-linked dominant condition due to de novo mutations in the MECP2 gene, whereas autism is a neurodevelopmental and behavioral disorder with complex genetic basis. Maternally inherited duplications on 15q11-q13 are found in a fraction of autistic children suggesting that an abnormal dosage of gene(s) within this region might cause susceptibility to autism. Now we show that three Rett patients are carriers of both a MECP2 mutation and a $15 q 11-q 13$ rearrangement, suggesting that there might be a relationship between autism-related genes and the MECP2 gene.
\end{abstract}

European Journal of Human Genetics (2004) 12, 682-685. doi:10.1038/sj.ejhg.5201198

Published online 7 April 2004

Keywords: autism; Rett syndrome; chromosome 15 rearrangements

\section{Introduction}

Autism (OMIM \# 209850) is a relatively common condition with an estimated prevalence of 1 in 2500. Autism spectrum disorders, are even more common with an estimated prevalence of 1.3 in 1000. This group of disorders, usually characterized by impairment in communication and restricted interests towards external stimuli, is causally heterogeneous; ${ }^{1,2}$ however, a strong genetic component in autism is indicated by an increased concordance rate in monozygotic versus dizygotic twins ${ }^{3,4}$ and a relative risk for siblings of idiopathic cases that is 75fold increased with respect to the prevalence in the general population. ${ }^{5}$ Several candidate loci have been so far identified on the basis of full genomewide screen for autism susceptibility loci. ${ }^{6,7}$ Chromosome $15 q 11-q 13$ has

*Correspondence: Dr F Gurrieri, Istituto di Genetica Medica, Università Cattolica del S. Cuore, Facoltà di Medicina, L.go F. Vito 1, 00168 Roma, Italy. Tel: + 396 30154927; Fax: + 396 3050031;

E-mail: fgurrieri@rm.unicatt.it

Received 20 October 2003; revised 13 January 2004; accepted 4 March 2004 become a good candidate region for autism, based on the finding of maternally derived duplications of this region in autistic patients. ${ }^{8-10}$

Rett syndrome (OMIM \# 312750) is a severe neurodevelopmental disorder characterized by early onset deceleration of development followed by regression in young girls. Inability to speak is a cardinal feature of Rett syndrome, ${ }^{11}$ but some girls, over the years, recover the ability to speak, improving at the same time the use of their hands. ${ }^{12,13}$ These patients are grouped together under the heading of preserved speech variant (PSV) of Rett syndrome. ${ }^{14}$ About $80 \%$ of Rett syndrome cases and $50 \%$ of PSV have a de novo mutation in the transcriptional silencer MECP2 gene, classifying Rett syndrome as a monogenic X-linked dominant disorder. ${ }^{15-18}$ Subsequently, it has been observed that MECP2 mutations may also result in mental retardation in males and are silent in related carrier females, ${ }^{19,20}$ suggesting a role of the genetic background in the determination of the phenotype.

Based on clinical overlap of Rett syndrome and autism, we hypothesized that there might be an interaction 
between MECP2 and genes on chromosome 15 and decided to investigate a group of Rett patients for the presence of $15 \mathrm{q} 11-\mathrm{q} 13$ rearrangements.

\section{Materials and methods Subjects}

We enrolled a total of 63 female Rett patients with a de novo MECP2 mutation. As far as the phenotypes are concerned we recognized 45 patients presenting with classic Rett syndrome, 14 with PSV, two with form fruste and two with Rett variants (criteria according to Trevathan and Naidu, ${ }^{11}$ Hagberg and Skejeldal ${ }^{21}$ and Zappella et al. ${ }^{22}$ )

\section{Microsatellite analysis}

Initially, we genotyped each patient and her parents with the following microsatellite markers located within the 15q11-q13 genomic region: D15S646, D15S817-copy 1, D15S817-copy 2 and D15S1021 (see Figure 1).

In order to obtain a more saturated map of microsatellite loci around markers D15S646 and D15S817-copy 1, we identified in the database (at http://genome.ucsc.edu) nine new microsatellite repeats (referred to as D15S1A, D15S2A, D15S3A, D15S4A, D15S5A, D15S6A, D15S7A, D15S8A, D15S9A) and designed primers to amplify each of them using the program OLIGO for MS-DOS (sequences are indicated in Table 1). A map of the microsatellite loci analyzed with the relative distances is reported in Figure 1. Alternative primers to amplify D15S646, indicated as D15S646bisF and D15S646bisR in Table 1, were tested in order to rule out the presence of null alleles at D15S646 due to primer polymorphism.

\section{Controls}

We analyzed 400 control chromosomes at locus D15S817copy 1 and 400 control chromosomes at locus D15S646. For D15S646, we followed the segregation of alleles in 200 trios composed by son-mother-father.

\section{Results}

We found a $15 \mathrm{q}$ rearrangement in three Rett patients out of 63: \#10, \#203 and \# 307.

\section{Clinical phenotypes}

Patient \# 10 and patient \# 203, now aged 7 and 12 years respectively, have the clinical manifestations of classic Rett syndrome. Regression is reported as occurring at age 12 months and both are presently in third stage, with typical stereotypic 'hand washing' activities, apraxia, absence of speech, mild scoliosis, stypsis and cold extremities, frequent hyperventilation and seizures. Patient \# 307, now aged 32 years, is the first reported case of PSV. ${ }^{14}$ She walked at 19 months and began to show hand-washing activities by the age of 3 years when she started to say short phrases.
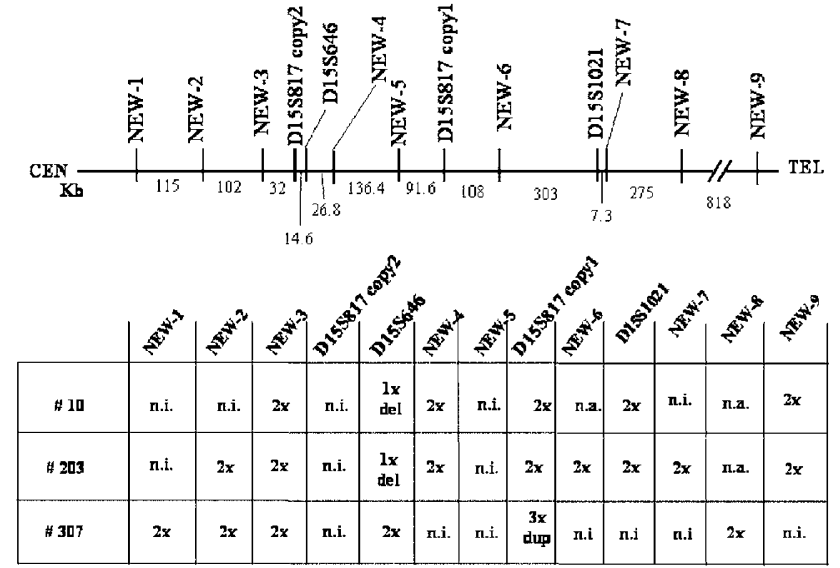

Figure 1 (Top) Physical map of the $15 q 11.2$ region with all microsatellite loci analyzed in Rett patients. For D15S817, we demonstrated that the published primers amplify two different genomic regions that we call here D15S817-copy 1 and D15S817-copy 2. In fact, a BlastN search using the 386 bp sequence of D15S817, reported in GDB, resulted in two hits in HTGS database (clones RP11 $350 A 1$ and RP11-682C24) that differ from each other by the presence of $11 \mathrm{bp}$ substitutions and a short sequence next to the TAGA repeat. In the aim to better distinguish between the two loci, we designed two new pairs of primers: 817FB2 (forward) and 817RB2 (reverse) for D15S817-copy 1 and 817FA2 (forward) and 817RA2 (reverse) for D15S817-copy 2. Analysis of these two couples of primers in 30 control trios showed that they amplified different alleles, which were transmitted in a Mendelian manner. Reciprocal distances are indicated in kb. (Bottom) List of the three Rett patients in which a $15 q 11.2$ rearrangement was detected. $2 x=$ normal number and segregation of alleles detected; $\mathrm{NI}=$ noninformanoninformative results; $\mathrm{DEL}=$ deletion; $\mathrm{DUP}=$ duplication; $\mathrm{NA}=$ not assessed.

In subsequent years, she had a partial improvement of her language and of the use of her hands. Her behavior has maintained the features of an autistic disorder.

\section{Marker analysis}

Results on these three patients are summarized in Figure 1. They carry a de novo MECP2 mutation that changes aminoacid R133. In two patients with classic Rett syndrome, we detected a deletion at D15S646 (Figure 1: patients \#10 and \#203), while in a patient with PSV we detected a duplication at D15S817-copy 1 (Figure 1: patient \#307). Analysis of 400 control chromosomes ruled out that duplication at D15S817-copy 1 was a polymorphism and genotyping of 200 control trios, as well as amplification of the same locus with alternative primers, ruled out that deletion at D15S646 was either a polymorphism or a null allele. Molecular analysis of markers surrounding D15S646 showed a normal segregation of alleles at loci D15S3A and D15S4A in patients \#10 and \#203, indicating that these loci 
Table 1 : List and sequences of the noncommercial primers used in patients \#10, \#203, and \#307

\begin{tabular}{ll}
\hline List & \multicolumn{1}{c}{ Sequence } \\
\hline $817 F B 2$ & TGGAACCAATAGGATAGACAC \\
$817 R B 2$ & GGTCAGCCTCCATAATCC \\
$817 F A 2$ & CAGAAGCAATAGGATAGATAT \\
$817 R A 2$ & GGTCAGCCTCCATAATCA \\
D15S1A F & AAATACCACACACACCCCACCAT \\
D15S1A R & AACCCAAACTCAACTCCACAGA \\
D15S2A F & AGCCTAGGCGACAGAGTCCGAT \\
D15S2A R & AGCTTGCAGTCAGACTAGATCGC \\
D15S3A F & ATGGTGCACGCCTGTAAAAAAAG \\
D15S3A R & GCCTATGATCCACCTGGAATTGA \\
D15S4A F & GCATCAGGAAGCCAACTAAATCA \\
D15S4A R & AAGGCTGACCATTTACACACAT \\
D15S5A F & GTATTTGTTTCTGCTCCTGAG \\
D15S5A R & ATCAATCTAAGTGCCCATCAACC \\
D15S6A F & GGTTTCTAGAGCAACAGAACGA \\
D15S6A R & AACATGCATCCCAGGATCTC \\
D15S7A F & TTGCCATGTAAGGTCTTCAGA \\
D15S7A R & CACTGCCCAGATCATTGTT \\
D15S8A F & TGGGTTGGCATGTCCTGAATATA \\
D15S8A R & ATAGGGAATGTGTTCCGTGTGTG \\
D15S9A F & CTCCAGAGAAACAGCAAGAATAGAAGAT \\
D15S9A R & CTAATCTATCCCTCCATCTACCTGTCAT \\
D15S646bisF & CTAGATGACGGGTTAGTGGGTG \\
D15S646bisR & TGCATGTGTGTTGTATATGTGTGTG \\
\hline
\end{tabular}

can be considered as the borders of the deletion, since D15S817-copy 2 was noninformative. Therefore, the critical interval deleted is comprised between D15S3A and D15S4A, which are located about $73 \mathrm{~kb}$ apart. Genotyping of patient \#307 and her family with markers surrounding D15S817-copy 1 was not helpful to define the extension of the duplication due to uninformativeness of markers. Quantitative studies will be necessary to overcome this problem.

Segregation analysis was extended to other family members in all three cases and indicated that the D15S817-copy 1 duplication was paternally inherited, while the D15S646 deletions were maternally inherited (Figure 2). The Rett patient \# 10 and her two healthy sisters inherited the D15S646 deletion from their mother. The two healthy girls did not carry an MECP2 mutation. The D15S646 deletion was inherited from the mother by the Rett patient \# 203 but not by her healthy sister, who also did not have an MECP2 mutation. The paternally inherited D15S817 duplication in patient \#307 arose in the gametes of the paternal grandfather.

\section{Discussion}

We and others have previously observed maternally inherited duplications at D15S817 locus in autistic patients. ${ }^{10,9,23,24}$ These findings suggest that an increased dosage of one or more maternally expressed genes or the a



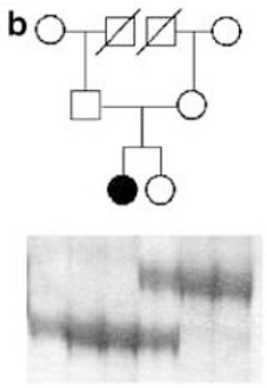

C

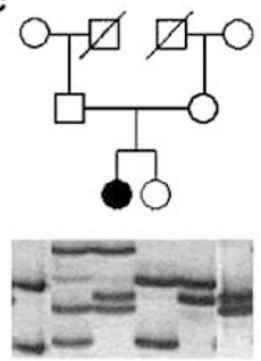

Figure 2 Segregation analysis of markers D15S646 and D15S817-1. In all, 6\% acrylamide gel of PCR product of marker D15S646 (panels a and b) and marker D15S817-1 (panel c). The pedigree of each family is drawn above the gel picture. (a) Family of proband \#10. The proband and the two sisters have only the paternal allele (lower band) and lack the maternal allele (upper band). (b) Family of proband \#203. The proband has only the paternal allele (lower band) and lacks the maternal allele (upper band). (c) Family of proband \# 307. The proband and the father have three alleles. The proband inherited the middle allele from the mother and the upper and lower alleles from the father. The paternal grandmother has two normal alleles.

lack of one or more paternally expressed genes might cause susceptibility to autism. Now we show that approximately $5 \%$ of Rett patients are carriers of both an MECP 2 mutation and a 15q11-q13 rearrangement. We excluded that such rearrangements represent polymorphisms. This frequency is higher than that reported in autistic patients, in which a duplication of chromosome $15 q 11-q 13$ is found in about $1 \%$ of cases. $^{23,24}$ Our results specifically point to the interval around markers D15S646 and D15S817-copy 1. This region seems to be a gene-poor region. NIX program analysis (http://www.hgmp.mrc.ac.uk/Registered/Webapp/ nix) of the sequence surrounding D15S817-copy 1 revealed the presence of an EST (EMBL HS8053) that is expressed in fetal brain. Further analysis is necessary to define whether this is a true gene.

We demonstrated that three Rett patients have a double molecular defect, one on MECP2 and the other in the $15 q 11-q 13$ region. A clear interpretation of these results is not yet possible because, even though it has been ruled out that the $15 q$ defect represents a polymorphism, still the phenotype of our patients with the double molecular defect is apparently not different from what is commonly observed in typical Rett syndrome or in the PSV subtype. Nevertheless, since the phenotypic expression of MECP2 mutations is influenced by the genetic background, we can hypothesize that the $15 \mathrm{q}$ rearrangements observed represent one of the genetic determinants of the clinical variability of Rett syndrome.

Interestingly, all three Rett patients are carriers of an MECP2 mutation at amino-acid R133. This position represents a hotspot for mutations in Rett patients; therefore, our observation of a $15 q$ rearrangement with a 
mutation at this specific site may well be due to chance. However, a larger number of patients needs to be studied to clarify this issue.

We observed that the Rett patients reported here have maternally inherited deletions and a paternally inherited duplication. Owing to the limited number of cases, this correlation with the sex of the transmitting parent and type of rearrangement may be due to chance. Alternatively, this correlation may reflect the underlying mechanism and could suggest the presence of genes imprinted in opposite sense near markers D15S646 and D15S817, similarly to what happens in the nearby Angelman/Prader-Willi region. Such genes might interact with MECP2.

\section{Acknowledgements}

This work was supported by Telethon Grant \# GGP02372A to FG and $A R$ and by Fondazione Rulfo, PAR 2002 University of Siena, FIRB 2001 \#RBAU015RN4 and by Ministero della Salute Ricerca Finalizzata 2003 grants to AR. We also thank Franca Cambi for the critical reading of the manuscript.

\section{References}

1 Ritvo ER, Mason Brothers A, Freeman BJ et al: The UCLAUniversity of Utah epidemiologic survey of autism: the etiologic role of rare diseases. Am J Psychiatry 1990; 147: 1614-1621.

2 Gillberg C, Coleman M: Autism and medical disorders: a review of the literature. Dev Med Child Neurol 1996; 38: 191-202.

3 Bailey A, Le Couteur A, Gottesman I et al: Autism as a strongly genetic disorder: evidence from a British twin study. Psychol Med 1995; 25: 63-77.

4 Steffenburg S, Gillberg C, Hellgren L et al: A twin study of autism in Denmark, Finland, Iceland, Norway and Sweden. J Child Psychol Psychiatr 1989; 30: 405-406.

5 Bolton P, Macdonald H, Pickles A et al: A case-control family history study of autism. J Child Psychol Psychiatry 1994; 35: 877-900.

6 International Molecular Genetic Study of Autism Consortium: A full genome screen for autism with evidence for linkage to a region on chromosome 7q. Hum Mol Genet 1998; 7: 571-578.

7 Philippe A, Martinez M, Guillod-Bataille M et al: Paris Autism Research International Sibpair Study. Genome-wide scan for autism susceptibility genes. Hum Mol Genet 1999; 8: 805-812.

8 Repetto GM, White LM, Bader PJ, Johnson D, Knoll JHM: Interstitial duplications of chromosome region 15q11-q13: clinical and molecular characterization. Am I Med Genet 1998; 79: 82-89.

9 Schroer RJ, Phelan MC, Michaelis RC et al: Autism and maternally derived aberrations of chromosome 15q. Am J Med Genet 1998; 76: $327-336$.

10 Gurrieri F, Battaglia A, Torrisi L et al: Pervasive developmental disorder and epilepsy due to maternally derived duplication of 15q11-q13. Neurology 1999; 52: 1694-1697.

11 Trevathan E, Naidu S: The clinical recognition and differential diagnosis of Rett syndrome. I Child Neurol 1988; 3 (Suppl): S6-S16.

12 Skjeldal OH, von Tetzchner S, Jacobsen K, Smith L, Heiberg A Rett syndrome, distribution of phenotypes with special attention to the preserved speech variant. Neuropediatrics 1995; 26: 87

13 Zappella M: The preserved speech variant of the Rett complex: a report of 8 cases. Eur Child Adolesc Psychiatry 1997; 6 (Suppl): $23-25$.

14 Zappella M: The Rett girls with preserved speech. Brain Dev 1992; 14: $98-101$.

15 Amir RE, Van den Veyver IB, Wan M, Tran CQ, Francke U, Zoghbi HY: Rett syndrome is caused by mutations in X-linked MECP2, encoding methyl-CpG-binding protein 2. Nat Genet 1999; 23: $185-188$.

16 De Bona C, Zappella M, Hayek G et al: Preserved speech variant as allelic of classic Rett syndrome. Eur J Hum Genet 2000; 8: 325-330.

17 Zappella M, Meloni I, Longo I, Hayek G, Renieri A: Preserved speech variant of the Rett syndrome: molecular and clinical dissection. Am J Med Genet 2001; 104: 14-22.

18 Zappella M, Meloni I, Longo I et al: Study of MECP2 gene in Rett syndrome variants and autistic girls. Am J Med Genet 2003; 119: 102-107.

19 Meloni I, Bruttini M, Longo I et al: A mutation in the Rett syndrome gene, MECP2, causes X-linked mental retardation and progressive spasticity in males. Am J Hum Genet 2000; 67: 982-985.

20 Couvert P, Bienvenu T, Aquaviva $\mathrm{C}$ et al: MECP2 is highly mutated in X-linked mental retardation. Hum Mol Genet 2001; 10: 941-946.

21 Hagberg BA, Skjeldal OH: Rett variants: a suggested model for inclusion criteria. Pediatr Neurol 1994; 11: 5-11.

22 Zappella M, Gillberg C, Ehlers S: The preserved speech variant: a subgroup of the Rett complex: a clinical report of 30 cases. $J$ Autism Dev Disord 1998; 28: 519-526.

23 Boyar FZ, Whitney MM, Lossie AC et al: A family with a grandmaternally derived interstitial duplication of proximal $15 \mathrm{q}$. Clin Genet 2001; 60: 421-430.

24 Wolpert CM, Menold MM, Bass MP et al: Three probands with autistic disorder and isodicentric chromosome 15. Am J Med Genet 2000; 96: 365-372. 